\title{
Editorial
}

\section{Applications of Polarimetric SAR}

\author{
Jian Yang, ${ }^{1}$ Yoshio Yamaguchi, ${ }^{2}$ Jong-Sen Lee, ${ }^{3}$ \\ Ridha Touzi, ${ }^{4}$ and Wolfgang-Martin Boerner ${ }^{5}$ \\ ${ }^{1}$ Department of Electronic Engineering, Tsinghua University, Beijing 100084, China \\ ${ }^{2}$ Department of Information Engineering, Niigata University, Niigata 950-2181, Japan \\ ${ }^{3}$ Computational Physics Inc., Springfield, VA 22151-2110, USA \\ ${ }^{4}$ Canada Centre for Remote Sensing, Natural Resources Canada, 588 Booth Street, Ottawa, ON, Canada K1A OY7 \\ ${ }^{5}$ UIC-ECE Communications, Sensing \& Navigation Laboratory, University of Illinois at Chicago, 900 W. Taylor Street, \\ SEL (607) W-4210, M/C 154, Chicago, IL 60607, USA
}

Correspondence should be addressed to Jian Yang; yangjian_ee@tsinghua.edu.cn

Received 21 May 2015; Accepted 27 May 2015

Copyright (c) 2015 Jian Yang et al. This is an open access article distributed under the Creative Commons Attribution License, which permits unrestricted use, distribution, and reproduction in any medium, provided the original work is properly cited.

Polarimetric SAR is an advanced imaging radar system; it plays an important role in radar remote sensing. With a polarimetric SAR, we can obtain much more information than conventional SAR systems (i.e., single polarized SAR systems) [1]. Up to now, various airborne and spaceborne polarimetric SAR systems have been developed, such as AirSAR, PI-SAR-1/2, E/F-SAR, CV-580 SAR, SIR-C/X-SAR, ALOS-PALSAR1/2, Radarsat-2, TerraSAR-X, and TanDEM$\mathrm{X}$. They have measured a huge mass of fully polarimetric data. Now polarimetric SAR has many applications in many fields, including agriculture (crop classification, soil moisture extraction, and crop assessment), oceanography (surface currents and wind field retrieval), forestry (forest monitoring, classification, and tree height estimation), disaster monitoring (oil spill detection, disaster assessment), and military (ship detection, target recognition/classification).

After calibration of the polarimetric SAR and image speckle filtering, feature extraction is the key step for target detection and target classification. Some important features were introduced, such as the polarization ratio and the polarization entropy [2]. An important approach to feature extraction is target decomposition. It is to decompose a scattering matrix or a covariance matrix to the linear combinations of some special typical scattering, such as the single bounce scattering, the double bounce scattering, and the volume scattering. The important decomposition includes
Krogager's decomposition [3], Cloude-Pottier's decomposition [2], Freeman-Durden's decomposition [4], Yamaguchi's decomposition [5], Touzi's decomposition [6], and CameronRais decomposition [7]. However, it is impossible to find a matrix to describe various volume scattering. So we still need to improve the volume scattering model in this approach. Another attempt to extract features is to use similarity between two matrices [8]. This method is independent of target decomposition and it can also be used to extract the features on the single bounce scattering, the double bounce scattering, and so on.

With target features, we can classify different kinds of targets/land covers. A lot of investigations have been made, for example, the complex Wishart distribution based method [9], target decomposition based methods [2-7], multifrequency SAR data fusion based methods, and quantitative comparison of classification capability of fully polarimetric versus dualand single-polarization SAR [10].

Damage monitoring is an important topic in remote sensing [11]. In this special issue, a paper is to investigate the temporal behavior of geometrical structural change of cropland affected by four different types of damages. The authors used a lot of polarimetric SAR data and optical time series data and made a lot of investigations.

Parameter estimation is another important topic in polarimetric SAR applications. Up to now, many investigations 
have been made in soil moisture extraction and tree height estimation. Cloude and Papathanassiou [12] made significant contribution to applications of polarimetric-interferometric SAR, especially to estimation of tree height. In this special issue, a paper is to investigate the impact of topography and tidal height by ALOS-1 measurements on $\mathrm{HH}$ and $\mathrm{HV}$ for estimating above ground biomass of mangrove forest in Indonesia. Another paper is to retrieve the depth of subsurface brine layer in Lop Nur by copolarized phase difference of surface scattering. From both papers, readers will find the potential ability of polarimetric SAR in quantitative remote sensing.

Compact polarimetric SAR is a special dual polarized SAR system. Comparing a polarimetric SAR system, compact polarimetric SAR has some advantages in pulse repeat frequency and width of surveying although it has some disadvantages. In this special issue, two papers are on the applications of compact polarimetric SAR. Readers will find that compact polarimetric SAR can be used for oil spill detection and classification.

"We are very fortunate to be at the doorstep of the golden age for developing polarimetric SAR applications." We will find more applications in the near future.

$$
\begin{array}{r}
\text { Jian Yang } \\
\text { Yoshio Yamaguchi } \\
\text { Jong-Sen Lee } \\
\text { Ridha Touzi } \\
\text { Wolfgang-Martin Boerner }
\end{array}
$$

distribution," International Journal of Remote Sensing, vol. 15, no. 11, pp. 2299-2311, 1994.

[10] J.-S. Lee and E. Pottier, Polarimetric Radar Imaging: From Basics to Applications, CRC Press, Boca Raton, Fla, USA, 2009.

[11] A. Gaber, F. Soliman, M. Koch, and F. El-Baz, "Using fullpolarimetric SAR data to characterize the surface sediments in desert areas: a case study in El-Gallaba Plain, Egypt," Remote Sensing of Environment, vol. 162, pp. 11-28, 2015.

[12] S. R. Cloude and K. P. Papathanassiou, "Polarimetric SAR interferometry," IEEE Transactions on Geoscience and Remote Sensing, vol. 36, no. 5, pp. 1551-1565, 1998.

\section{References}

[1] W.-M. Boerner, "Recent advances in extra-wide-band polarimetry, interferometry and polarimetric interferometry in synthetic aperture remote sensing and its applications," IEE Proceedings: Radar, Sonar and Navigation, vol. 150, no. 3, pp. 113124, 2003.

[2] S. R. Cloude and E. Pettier, "A review of target decomposition theorems in radar polarimetry," IEEE Transactions on Geoscience and Remote Sensing, vol. 34, no. 2, pp. 498-518, 1996.

[3] E. Krogager, "New decomposition of the radar target scattering matrix," Electronics Letters, vol. 26, no. 18, pp. 1525-1527, 1990.

[4] A. Freeman and S. L. Durden, "A three-component scattering model for polarimetric SAR data," IEEE Transactions on Geoscience and Remote Sensing, vol. 36, no. 3, pp. 963-973, 1998.

[5] Y. Yamaguchi, T. Moriyama, M. Ishido, and H. Yamada, "Four-component scattering model for polarimetric SAR image decomposition," IEEE Transactions on Geoscience and Remote Sensing, vol. 43, no. 8, pp. 1699-1706, 2005.

[6] R. Touzi, "Target scattering decomposition in terms of rollinvariant target parameters," IEEE Transactions on Geoscience and Remote Sensing, vol. 45, no. 1, pp. 73-84, 2007.

[7] W. L. Cameron and H. Rais, "Conservative polarimetric scatterers and their role in incorrect extensions of the Cameron decomposition," IEEE Transactions on Geoscience and Remote Sensing, vol. 44, no. 12, pp. 3506-3516, 2006.

[8] J. Yang, Y.-N. Peng, and S.-M. Lin, "Similarity between two scattering matrices," Electronics Letters, vol. 37, no. 3, pp. 193194, 2001.

[9] J.-S. Lee, M. R. Grunes, and R. Kwok, "Classification of multilook polarimetric SAR imagery based on complex Wishart 

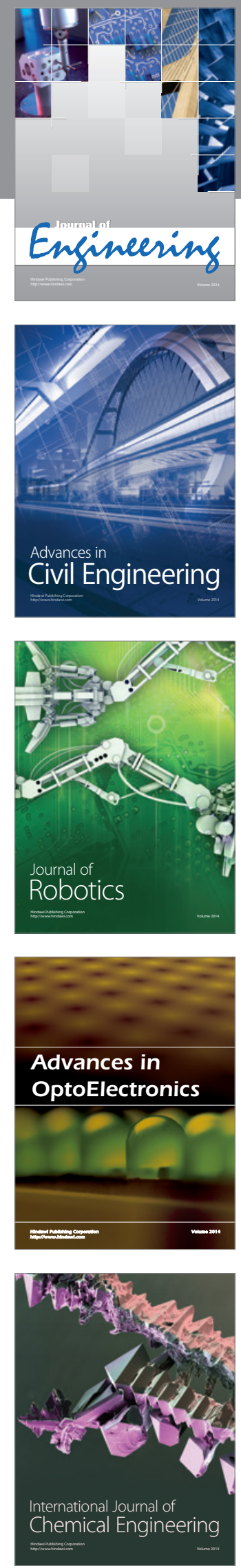

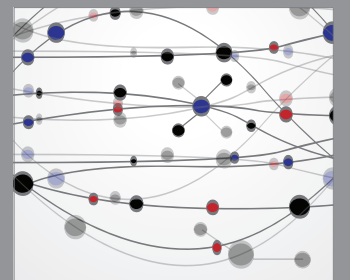

The Scientific World Journal
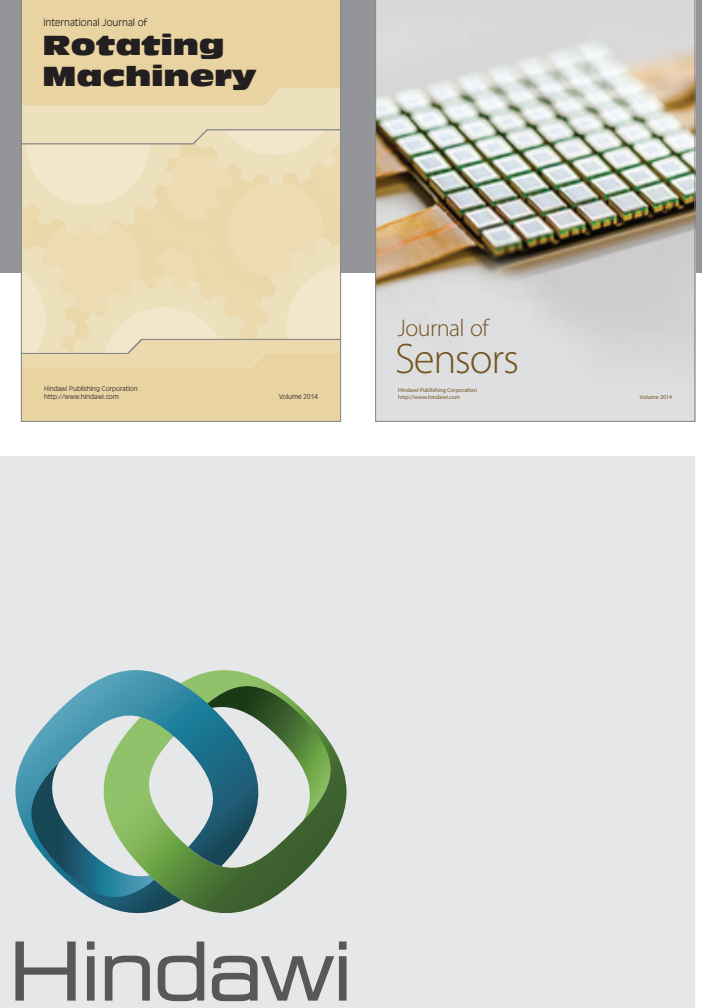

Submit your manuscripts at http://www.hindawi.com
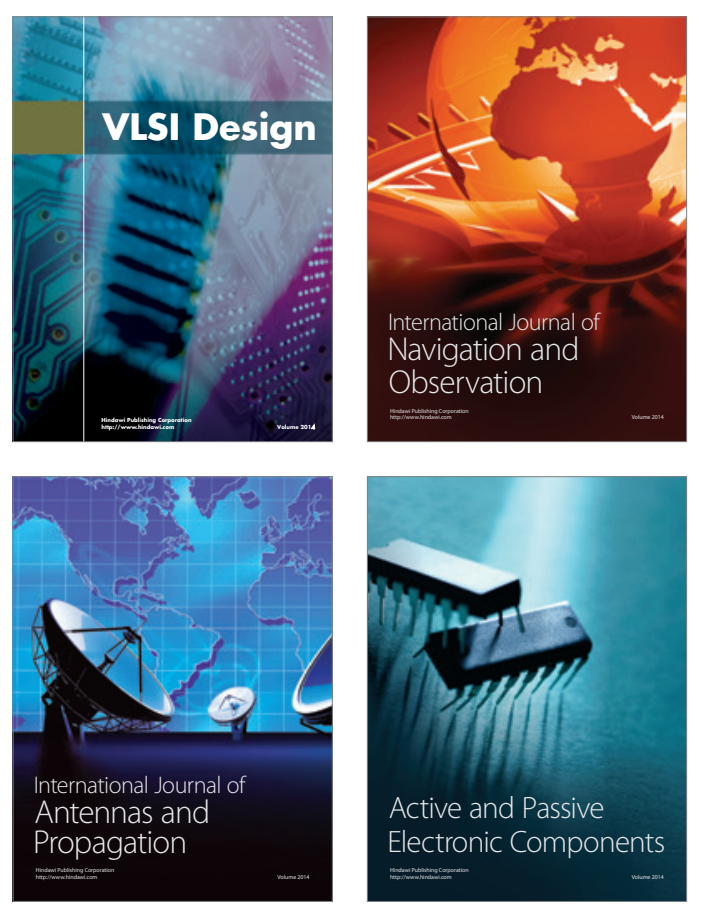
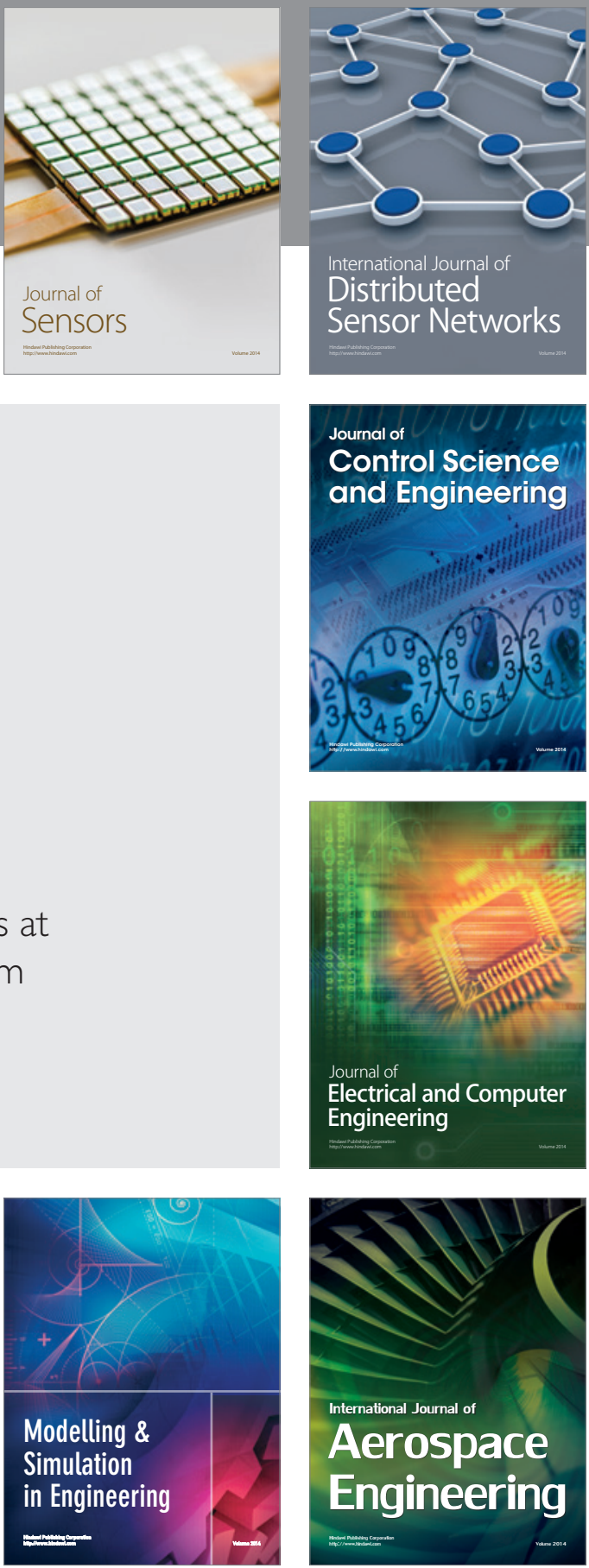

Journal of

Control Science

and Engineering
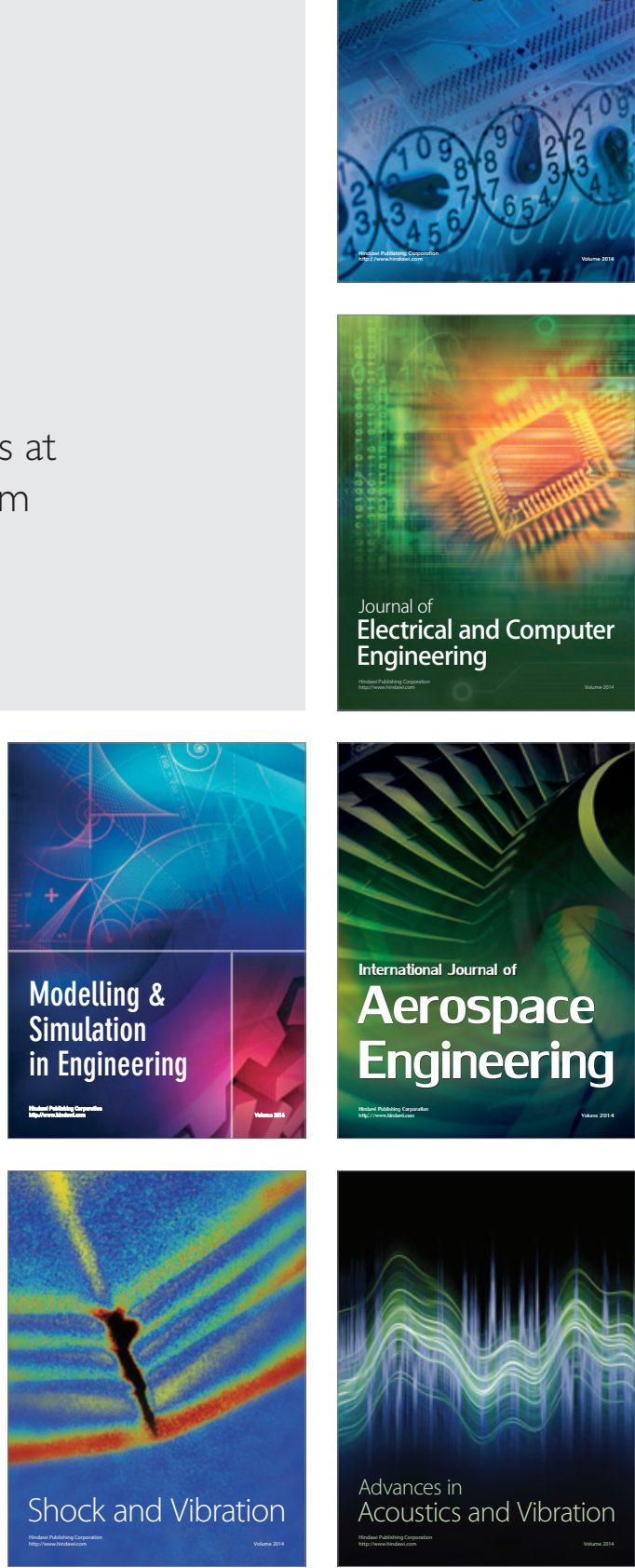